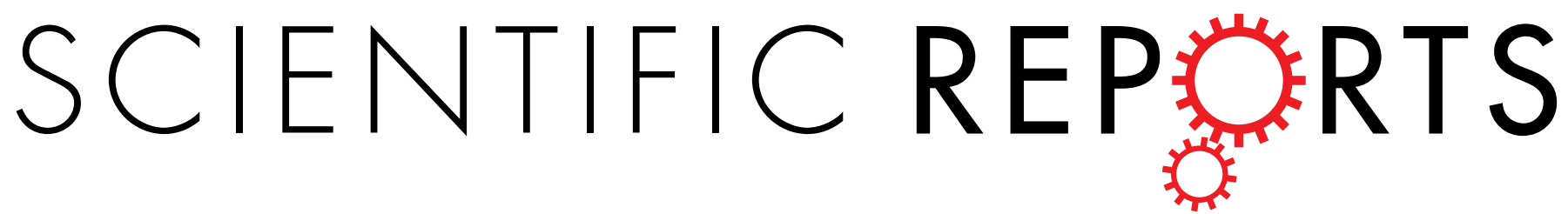

\title{
Corrigendum: Chromatic acclimation and population dynamics of green sulfur bacteria grown with spectrally tailored light
}

Semion K. Saikin, Yadana Khin, Joonsuk Huh, Moataz Hannout, Yaya Wang, Farrokh Zare, Alán Aspuru-Guzik \& Joseph Kuo-Hsiang Tang

Scientific Reports 4:5057; doi: 10.1038/srep05057; published online 27 May 2014; updated on 13 July 2015 In this Article, Figure $4 \mathrm{e}$ is a duplication of Figure $4 \mathrm{~d}$. The correct Figure $4 \mathrm{e}$ appears below as Figure 1.

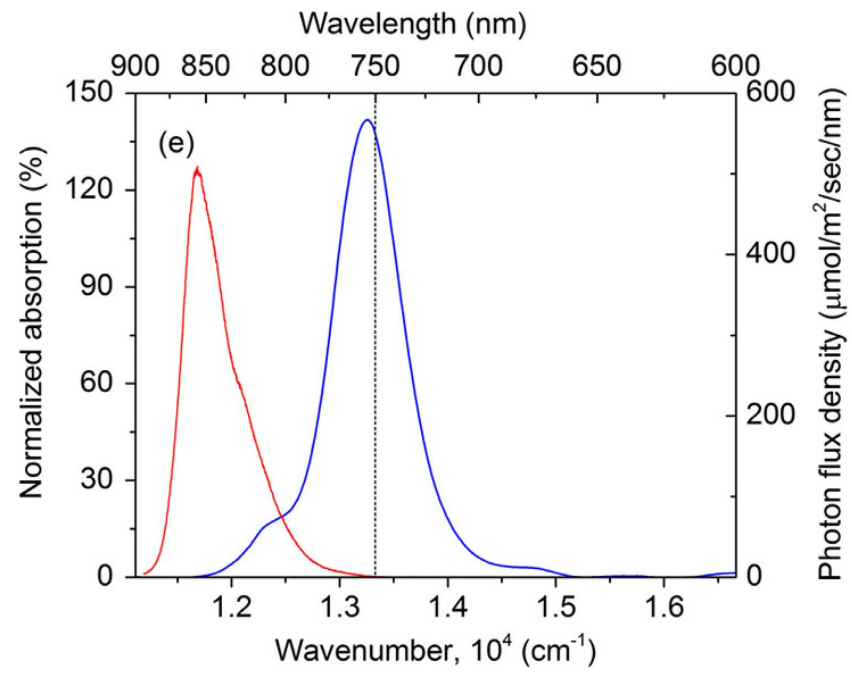

Figure 1. 